\title{
PUNÇÃO ASPIRATIVA POR AGULHA FINA NO DIAGNÓSTICO DE LINFADENOPATIAS E TUMORES SÓLIDOS EM CRIANÇAS E ADOLESCENTES
}

\author{
FINE NEEDLE ASPIRATION BIOPSY IN THE DIAGNOSIS OF LYMPHADENOPATHIES \\ AND SOLID TUMORS IN CHILDREN AND ADOLESCENTS
}

\author{
Wallace Acioli Freire de Gois ${ }^{1}$; Carlos Augusto Almeida Correa²; \\ Elaine Maria de Oliveira Alves, TCBC-DF ${ }^{3}$; Paulo Tubino, ECBC-DF ${ }^{4}$
}

\begin{abstract}
RESUMO: Objetivo: Avaliar a importância diagnóstica da punção aspirativa por agulha fina (PAAF) em crianças e adolescentes portadores de linfadenopatias e tumores sólidos. Métodos: Análise retrospectiva dos pacientes com idade igual ou inferior a 18 anos, atendidos no Centro de Pediatria Cirúrgica do Hospital Universitário de Brasília, Universidade de Brasília, no período de julho de 2000 a julho de 2005. Foram calculados: sensibilidade, especificidade, precisão (acurácia) diagnóstica, valor preditivo positivo e valor preditivo negativo. Resultados: Em 50 pacientes estudados, os resultados da PAAF mostraram sensibilidade de $90,9 \%$; especificidade de $100 \%$, precisão diagnóstica de $95 \%$ e valores preditivos positivo e negativo de $100 \%$ e $90 \%$, respectivamente Conclusão: Em nossa experiência, a PAAF é um método preciso, simples e seguro, útil no diagnóstico de linfadenopatias e tumores sólidos em crianças e adolescentes (Rev. Col. Bras. Cir. 2008; 35(1): 005-008).
\end{abstract}

Descritores: Biópsia por agulha fina; Doenças linfáticas; Diagnóstico diferencial; Neoplasias

\section{INTRODUÇÃO}

A punção aspirativa por agulha fina (PAAF) é uma técnica antiga, preconizada desde o século XIX, mas que permaneceu pouco difundida pelo alto índice de complicações relacionadas ao diâmetro exagerado das agulhas usadas na época ${ }^{1}$. Agulhas verdadeiramente finas só foram utilizadas a partir da década de $1950^{2}$.

Progressivamente, o uso da PAAF como meio diagnóstico vem se ampliando pelas vantagens que apresenta, já que é um método simples, rápido, seguro e de baixo custo. É uma técnica de fácil aprendizado, que permite a utilização de métodos auxiliares como imunocitoquímica, análise citogenética convencional, microscopia eletrônica e citometria de fluxo $^{3-9}$. A PAAF está indicada em qualquer massa visualizável ou palpável, mas também pode ser aplicada em lesões profundamente situadas, desde que guiada por ultrasonografia, tomografia computadorizada ou outro método de diagnóstico por imagem ${ }^{10,11}$.

Em pacientes pediátricos, a PAAF tem sido aceita mais lentamente do que em adultos ${ }^{12,13}$. No entanto, na atualidade, o tratamento dos tumores sólidos malignos mais comuns da criança pode apresentar melhores resultados com o uso de quimioterapia neo-adjuvante, tornando a importância do método evidente. Por outro lado, neste grupo etário o tra- tamento dos linfomas é basicamente quimioterápico, sendo desnecessária qualquer intervenção cirúrgica para esta finalidade desde que se obtenha o diagnóstico microscópico.

Pela relevância da identificação precoce das doenças malignas, o objetivo do presente trabalho foi avaliar a validade diagnóstica da PAAF em crianças e adolescentes portadores de linfadenopatias e tumores sólidos, atendidos no Centro de Pediatria Cirúrgica do HUB/UnB.

\section{MÉTODO}

Foram estudados, retrospectivamente, os pacientes com idade igual ou inferior a 18 anos de idade submetidos à PAAF para esclarecimento diagnóstico no Centro de Pediatria Cirúrgica do HUB/UnB, no período de julho de 2000 a julho de 2005. Os pacientes foram caracterizados segundo idade e gênero.

Foram excluídos da pesquisa os pacientes: cujos responsáveis não assinaram o termo de consentimento livre e esclarecido; com informações imprecisas no prontuário; em que a PAAF foi realizada por outra equipe médica.

Todas as punções aspirativas por agulha fina foram feitas na enfermaria do Centro de Pediatria Cirúrgica do HUB. Para as punções foram usadas agulhas descartáveis $25 \times 6 \mathrm{~mm}$ acopladas a uma seringa de $20 \mathrm{~mL}$. Todas as coletas foram

\footnotetext{
1. Cirurgião Pediatra da Secretaria de Saúde do Distrito Federal; Professor Substituto da Área de Medicina da Criança e do Adolescente da Faculdade de Medicina/UnB.

2. Médico da Secretaria de Saúde do Distrito Federal.

3. Professora Associada da Área de Medicina da Criança e do Adolescente da Faculdade de Medicina/UnB; Chefe do Serviço de Oncologia Pediátrica do Centro de Pediatria Cirúrgica do HUB/UnB.

4. Professor Emérito da Universidade de Brasília; Chefe do Centro de Pediatria Cirúrgica do HUB/UnB.

Recebido em 14/08/2007

Aceito para publicação em 16/07/2007

Conflito de interesses: nenhum

Fonte de financiamento: nenhuma

Trabalho realizado no Centro de Pediatria Cirúrgica do Hospital Universitário de Brasília (HUB), Universidade de Brasília (UnB).
} 
realizadas pela equipe da Pediatria Cirúrgica do HUB utilizando técnica previamente descrita ${ }^{7}$. Em cada procedimento foram preparadas seis lâminas, imediatamente imersas em álcool a $70^{\circ}$ para evitar dessecamento do material. Era aspirado álcool a $70^{\circ}$ na agulha e seringa utilizadas. Em seguida, o material era encaminhado para o Centro de Anatomia Patológica do HUB (CAP-HUB), onde as lâminas foram coradas pelo método de Papanicolaou e o conteúdo da seringa centrifugado para confecção do bloco celular. Todo material coletado foi interpretado por citopatologista do CAP-HUB. As punções com resultados inicialmente insatisfatórios foram refeitas, utilizando-se a mesma técnica.

Para avaliação do método e determinação do valor preditivo da PAAF, os diagnósticos citológicos foram comparados com os diagnósticos histológicos dos mesmos tumores após a operação, nos casos em que foi feito o procedimento cirúrgico. No caso de paciente não operado, o acerto ou não da PAAF foi avaliado pelo acompanhamento clínico do mesmo.

Os achados da PAAF foram classificados em três categorias: malignos, benignos e inconclusivos. Quando o diagnóstico citológico foi inconclusivo apesar de mais de uma amostra, o mesmo foi alocado em grupo separado, sendo registrado o diagnóstico obtido pela histopatologia.

Foram utilizados testes de sensibilidade, especificidade, validade diagnóstica e valores preditivos positivo e negativo. Sensibilidade foi definida como VP/VP+FN em que VP é o número de verdadeiros positivos para malignidade e FN representa o número de falsos negativos para malignidade; especificidade é VN/VN+FP em que VN é o número de verdadeiros negativos para malignidade e FP o número de falsos positivos para malignidade; validade ou precisão ("acurácia") do método foi definida como VP+VN/ $\mathbf{V P + F N + F P + V N}$. Os valores preditivos positivo e negativo foram definidos, respectivamente, como VP/VP+FP e VN/ $\mathbf{V N}+\mathbf{F N}^{14}$

As normas éticas para pesquisa científica em seres humanos foram rigorosamente observadas e a pesquisa foi aprovada pelo Comitê de Ética em Pesquisa da Faculdade de Medicina/UnB.

\section{RESULTADOS}

Cento e setenta e cinco pacientes com idade igual ou inferior a 18 anos foram submetidos à PAAF no período estudado. Destes, em função dos critérios de exclusão, foram avaliados 50 pacientes, dos quais 27 (54\%) eram do gênero femi- nino e 23 (46\%) do gênero masculino. A média de idade foi cinco anos e nove meses (variando de três meses a 18 anos). Os locais anatômicos dos quais os espécimens foram colhidos estão relacionados na Tabela 1 ; em $50 \%$ dos casos o material foi obtido das regiões cervicais e da cabeça.

Os achados citológicos iniciais foram classificados como malignos em 20 pacientes $(40 \%)$ e benignos em outros $20(40 \%)$. Nos 10 casos restantes (20\%), o exame foi considerado inconclusivo a despeito de uma nova punção. A correlação entre os resultados da PAAF e os achados histológicos está detalhada na Tabela 2. Em um dos casos, cujo diagnóstico foi linfoma não-Hodgkin, após o início da quimioterapia específica houve regressão completa do tumor e a comprovação do resultado da PAAF foi obtida por meio do seguimento clínico. APAAF fez a diferenciação entre benignos e malignos em 38 dos 40 casos em que o exame foi conclusivo, com precisão diagnóstica de $95 \%$. Os demais testes evidenciaram sensibilidade de $90,9 \%$, especificidade de $100 \%$, valor preditivo positivo de $100 \%$ e valor preditivo negativo de $90 \%$.

\section{DISCUSSÃO}

Em nosso país, os dados referentes ao uso da PAAF na infância e na adolescência ainda são relativamente escassos e, mais comumente, relacionados a doenças da tireóide ${ }^{15-}$ ${ }^{18}$. No presente estudo foi avaliada a validade diagnóstica da PAAF em crianças e adolescentes portadores de linfadenopatias e tumores sólidos atendidos no Centro de Pediatria Cirúrgica do Hospital Universitário de Brasília.

Nos pacientes com linfadenomegalia o procedimento foi indicado apenas naqueles com aumento progressivo do linfonodo por duas a três semanas; nos que não apresentaram diminuição dos linfonodos após seis semanas de evolução ou que não retornaram ao tamanho normal após 10 a 12 semanas. A PAAF foi mais precoce quando os linfonodos aumentados eram supraclaviculares e também e a radiografia simples do

Tabela 1 - Locais das punções aspirativas por agulha fina.

\begin{tabular}{|c|c|}
\hline Locais puncionados & Número de pacientes (\%) \\
\hline Regiões cervicais e da cabeça & $25(50)$ \\
\hline Tórax & $6(12)$ \\
\hline Abdome & $14(28)$ \\
\hline Pelve & 3 (6) \\
\hline Outros & 2 (4) \\
\hline Total & $50(100)$ \\
\hline
\end{tabular}

Tabela 2 - Correlação entre os resultados da PAAF e os achados histológicos elou seguimento clínico.

\begin{tabular}{|c|c|c|c|c|}
\hline \multirow{3}{*}{$\begin{array}{l}\text { Resultados } \\
\text { daPAAF } \\
(n=50)\end{array}$} & & \multicolumn{3}{|c|}{ Comprovação } \\
\hline & & \multirow{2}{*}{$\begin{array}{c}\text { Seguimento clínico } \\
\text { Maligno }(n=1)\end{array}$} & \multicolumn{2}{|c|}{ Exame histológico } \\
\hline & & & Maligno $(n=23)$ & Benigno $(n=26)$ \\
\hline Maligno & 20 & 1 & 19 & 0 \\
\hline Benigno & 20 & 0 & 2 & 18 \\
\hline Inconclusivo & 10 & 0 & 2 & 8 \\
\hline
\end{tabular}


tórax apresentasse alguma anormalidade ${ }^{19}$. Com esta conduta, foi encontrada benignidade em apenas $40 \%$ dos nossos casos enquanto que outros autores, que utilizaram a PAAF como forma de triagem nas linfadenopatias, obtiveram diagnósticos de doenças benignas em $97 \%$ dos pacientes estudados ${ }^{20}$. Não recomendamos a PAAF como método de triagem, antes que se faça um estudo clínico e laboratorial minucioso. Embora com baixo índice de complicações, é um procedimento que só deve ser indicado de modo a trazer benefício aos pacientes, especialmente em se tratando de crianças.

Setenta e oito por cento das punções foram feitas em cabeça e pescoço (50\%) e abdômen (28\%). Esses locais também foram os mais frequientes em outros trabalhos ${ }^{4,5}$. Material insatisfatório foi obtido em $20 \%$ dos casos, taxa superior à de outras séries que apresentam resultados de $9 \%$ a $15 \%{ }^{13}$. Provavelmente isto se deve ao fato de que o Centro de Pediatria Cirúrgica do HUB é um centro de referência para a região, para o qual convergem doenças raras. Em um dos casos desta série, um tumor neuroectodérmico melanocítico da infância (em que há cerca de 378 casos descritos na literatura, até o momento), ${ }^{21}$ o diagnóstico citológico foi inconclusivo, embora existissem células típicas na amostra, e apenas quando correlacionado com o exame histopatológico foi reconhecida a citologia característica do tumor. As outras causas foram material insuficiente ou hemorrágico e erros na preparação das lâminas. Assim, deve ser enfatizado que além da disponibilidade de um citopatologista experiente para analisar as lâminas, são igualmente importantes a técnica de aspiração e a preparação adequada do esfregaço. Já há uma tendência em se fazer o exame citológico rapidamente, logo após o procedimento, enquanto o paciente aguarda, para que se possa determinar a celularidade da amostra. Caso esta seja insatisfatória, faz-se uma nova punção, diminuindo assim os resultados inconclusivos por celularidade insuficiente. Este procedimen- to nos parece promissor já que o exame citológico rápido mostra concordância com o tardio ${ }^{22}$.

Na presente pesquisa, os resultados da PAAF foram comparados com os do exame histopatológico em $98 \%$ dos casos e no único caso sem comprovação histopatológica a confirmação diagnóstica foi possível pelo seguimento clínico e resposta ao tratamento, dando confiabilidade aos resultados obtidos. Em alguns trabalhos a confirmação histopatológica não chega a $50 \% \%^{5,13}$.

Em nossa experiência, a PAAF de massas superficiais em crianças não é mais dolorosa ou traumática do que uma coleta de sangue e, geralmente, não há necessidade de sedação. Se houver disponibilidade, pode ser feita anestesia tópica da pele com uma emulsão óleo/água de lidocaína e prilocaína na proporção de 1:1 sob a forma de creme. Em nossos casos não houve complicações precoces ou tardias decorrentes do procedimento, comprovando a segurança da PAAF em crianças, o que foi evidenciado também por outros autores $^{5,7,13,23}$. Destacamos que não houve nenhum caso de implante tumoral no trajeto da punção.

Correlacionando-se os resultados citológicos com os histopatológicos, foram observadas sensibilidade de $90,9 \%$ e especificidade de $100 \%$, o que consideramos um bom resultado em comparação com a literatura científica mundial. A diminuição do valor da sensibilidade foi causada pelos dois resultados falso-negativos, mostrando que a PAAF tem maior valor diagnóstico quando associada a uma observação clínica criteriosa dos pacientes e a um bom seguimento daqueles com diagnóstico de lesão benigna, por três a seis meses.

Em conclusão, a PAAF é um método diagnóstico preciso, simples, minimamente invasivo e seguro na nossa experiência, útil para a avaliação inicial das massas benignas e malignas de partes moles na criança e no adolescente.

\begin{abstract}
Background: To evaluate the diagnostic value of the fine needle aspiration biopsy in children and adolescents with solid tumors or lymphadenopathy. Methods: 18 years old or less patients were observed since July 2000 to July 2005. We observed: sensibility, specificity, diagnostic accuracy and predictive value. Results: In 50 studied patients the fine needle aspiration biopsy we found $90.9 \%$ sensibility, 100\% specificity, 95\% diagnostic accuracy, 100\% positive predictive value and $90 \%$ negative predictive value. Conclusion: The fine needle aspiration biopsy is a easy, safe and accurate diagnostic method for solid tumors and lymphadenopathies.
\end{abstract}

Key words: Biopsy, Fine-Needle; Lymphatic diseases; Diagnosis, differential; Neoplasms.

\section{REFERÊNCIAS}

1. Ansari NA, Derias NW. Origins of fine needle aspiration cytology. J Clin Pathol. 1997;50(7):541-3.

2. Soderstrom N. Puncture of goiters for aspiration biopsy. Acta Med Scand. 1952;144(3):235-44.

3. Antley CM, Mooney EE, Layfield LJ. A comparison of accuracy rates between open biopsy, cutting-needle biopsy, and fine-needle aspiration biopsy of the breast: a 3-year experience. Breast $\mathbf{J}$. 1998;4(1):3-8.
4. Dey P, Mallik MK, Gupta SK, Vasishta RK. Role of fine needle aspiration cytology in the diagnosis of soft tissue tumours and tumour-like lesions. Cytopathology. 2004;15(1):32-7.

5. Palacios González RV, Peña Alonso R, Ramón García G, Cabrera Muñoz L, Sadowinski Pine S, Valencia Mayoral P, Salgado Jiménez A, Ontiveros Nevarez P. Comparación de la biopsia por aspiración con aguja fina y la biopsia quirúrgica en el diagnóstico de tumores en niños. Bol Med Hosp Infant Mex. 1997;54(7):305-10.

6. Masood S. Core needle biopsy versus fine needle aspiration biopsy: are there similar sampling and diagnostic issues? Clin Lab Med. 2005;25(4):679-88. 
7. Silverman JF, Gurley AM, Holbrook CT, Joshi VV. Pediatric fine-needle aspiration biopsy. Am J Clin Pathol. 1991;95(5):6539.

8. Schmitt F, Tani E, Tribukait B, Skoog L. Assessment of cell proliferation by Ki-67 staining and flow cytometry in fine needle aspirates (FNAs) of reactive lymphadenitis and non-Hodgkin's lymphomas. Cytopathology. 1999;10(2):87-96.

9. Drut R, Drut RM, Pollono D, Tomarchio S, Ibáñez O, Urrutia A, Ripoll MC. Fine-needle aspiration biopsy in pediatric oncology patients: a review of experience with 829 patients ( 899 biopsies). J Pediatr Hematol Oncol. 2005;27(7):370-6.

10. Sklair-Levy M, Lebensart PD, Applbaum YH, Ramu N, Freeman A, Gozal D, Gross E, Sherman Y, Bar-Ziv J, Libson E. Percutaneous image-guided needle biopsy in children — summary of our experience with 57 children. Pediatr Radiol. 2001;31(10):732-6.

11. Erturk SM, Mortelé KJ, Tuncali K, Saltzman JR, Lao R, Silverman SG. Fine-needle aspiration biopsy of solid pancreatic masses: comparison of CT and endoscopic sonography guidance. AJR Am J Roentgenol. 2006;187(6):1531-5.

12. Howell LP, Russell LA, Howard PH, Teplitz RL. Fine needle aspiration biopsy of superficial masses in children. West J Med. 1991;155(1):33-8.

13. Orford JE, Gollow I, Brennan B, Hallam L. Fine needle aspiration biopsy in children. Aust N Z J Surg. 1997;67(11):785-8

14. Pereira MG. Aferição dos efeitos. In: Pereira MG. Epidemiologia: teoria e prática. Rio de Janeiro: Guanabara Koogan; 1995. p. 359-76.

15. Bisi H, Camargo RY, Fernandes VS, Bloise W, Longatto Filho A. Citologia aspirativa de tireóide por agulha fina em crianças e adolescentes. J Pediatr (RJ). 1990;66(10/12):247-50.

16. Setian N, Manna TD, Damiani D, Dichtchekenian V. O bócio na faixa etária pediátrica: implicações diagnósticas e terapêuticas. J Pediatr (RJ). 1991;67(1/2):69-73.

17. Biazotto JR, Duarte Jr. S, Alves EMO, et al. Punção aspirativa por agulha fina em tumores sólidos em crianças. In: SOBOPE.
Resumos Científicos do X Congresso da Sociedade Latino-Americana de Oncologia Pediátrica. IV Congresso da Sociedade Brasileira de Oncologia Pediátrica. II Jornada de Cirurgia Oncológica Pediátrica da SOBOPE; 1993 Out 24-28; Rio de Janeiro, RJ; 1993. p.95.

18. Barros MAE, Borges MF, Lima MA, Ferreira BP, Pelegrinelli AC, Santos TG. Tireoidite crônica na infância e adolescência. Arq Bras Endocrinol Metab. 1994;38(2):96-9.

19. Tubino P, Alves E. Pediatria cirúrgica: diagnóstico e tratamento. Brasília: Universidade de Brasília; 2003.

20. Handa U, Mohan H, Bal A. Role of fine needle aspiration cytology in evaluation of paediatric lymphadenopathy. Cytopathology. 2003;14(2):66-9.

21. Fowler DJ, Chisholm J, Roebuck D, Newman L, Malone M, Sebire NJ. Melanotic neuroectodermal tumor of infancy: clinical, radiological, and pathological features. Fetal Pediatr Pathol. 2006;25(2):59-72.

22. Nasuti JF, Gupta PK, Baloch ZW. Diagnostic value and costeffectiveness of on-site evaluation of fine-needle aspiration specimens: review of 5,688 cases. Diagn Cytopathol. 2002;27(1):1-4.

23. Al-Shaikh A, Ngan B, Daneman A, Daneman D. Fine-needle aspiration biopsy in the management of thyroid nodules in children and adolescents. J Pediatr. 2001;138(1):140-2.

Como citar este artigo:

Góis WA, Correa CA, Alves EM, Tubino PJ. Punção aspirativa por agulha fina no diagnóstico de linfadenopatias e tumores sólidos em crianças e adolescentes. Rev Col Bras Cir. [periódico na Internet] 2008; 35(1). Disponível em URL: http://www.scielo.br/rcbc

Endereço para correspondência:

Elaine Maria de Oliveira Alves

SHIN QI-2 CJ.6 Casa 4 - Lago Norte

71510-060 - Brasília - DF

E-mail: emo-alves@uol.com.br 